\title{
Design, synthesis and antimicrobial evaluation of s-triazinyl urea and thiourea derivatives
}

\author{
Pankaj B. Kaswala,* Kishor H. Chikhalia, Nisha K. Shah, Dhaval P. Patel, Dharmendra H. \\ Patel and Govindraj V. Mudaliar \\ Department of Chemistry, School of Sciences, Gujarat University, \\ Ahmedabad-380 009, Gujarat, India \\ E-mail: pankajkaswala@gmail.com
}

\begin{abstract}
A series of urea and thiourea derivatives of s-triazine have been developed based on high yielding nucleophilic substitution of 2,4,6-trichloro-1,3,5-triazine by 4-hydroxy coumarin, cyclopropylamine and ammonia at suitable conditions. These were further treated with various substituted aryl isocyanate and aryl isothiocyanate. All the synthesized compounds were evaluated for their antibacterial activities against various Gram-positive and Gram-negative strains of bacteria. A few compounds showed good to superior in vitro antibacterial activity against S.aureus, B.subtilis, E.coli and P.aeruginosa respectively. The new synthesized compounds were characterized using IR, ${ }^{1} \mathrm{H}-\mathrm{NMR}$ and elemental analysis.
\end{abstract}

Keywords: s-Triazine, urea, thiourea, 4-hydroxy coumarin, antibacterials

\section{Introduction}

The exploration of heterocycles as privileged structures in drug discovery is, beyond doubt, one of the major areas in medicinal chemistry. These privileged structures represent a class of molecules that act as ligands for various biological receptors with a high degree of binding affinity. Problems of multi-drug resistant microorganisms have reached on alarming level in many countries around the world. A numbers of recent clinical reports describe the increasing occurrence of meticillin-resistant $S$. aureus and other antibiotic-resistant human pathogenic microorganisms in United State and European countries. Infections caused by those microorganisms pose a serious challenge to the medical community and the need for an effective therapy has led to a search for novel antimicrobial agents. Exploitation of these molecules should allow us to rapidly discover new biologically active compounds across a broad range of therapeutic areas in a shorter time scale. 
In this work, we report the synthesis and biological activity of some 1,3,5-triazinyl urea and thiourea analogues a class of privileged structures that have a wide range of biological properties. Among the compound having good antimicrobial properties, s-triazine derivatives constitute an important class of compounds possessing diverse pharmacological activities including broadly active as herbicidal ${ }^{1}$ and antimicrobial. ${ }^{2}$ Some are also used for the treatment of HIV infection. ${ }^{3-5}$ Several workers investigated the s-triazine nucleus in the scope of potential therapeutic agents for diseases due to bacteria ${ }^{6-9}$ cancer, ${ }^{10-12}$ antitumor ${ }^{13-14}$ and malaria. ${ }^{15-16}$ The above literature survey led us to consider the s-triazine nucleus as a possible scaffold.

Coumarins constitute an important class of compounds with several types of pharmaceutical agents possessing anticancer, anti-HIV, anticoagulant and antiproliferative activity. ${ }^{17}$ 4-Hydroxy coumarin has specific space among other coumarin derivatives, it possess anticonvulsant, antibacterial, antifungal and antitumor activities. ${ }^{18-19}$

In the design of new compounds, development of hybrid molecules through the combination of different pharmacophores in one structure may lead to compounds with increased antimicrobial activity. Therefore, these observations prompted us to synthesize new s-triazine derivatives which were attached with coumarin ring through an oxygen bridge. Then, the synthesized compounds were tested against two Gram-positive bacteria (Staphylococcus aureus, Bacillus subtilis), two Gram-negative bacteria (Pseudomonas aeruginosa, Escherichia coli) using the broth microdilution method.

\section{Results and Discussion}

\section{Materials and Methods}

All reagents were of analytical grade and use directly. All the melting points were determined in open glass capillary and are uncorrected. Progress of reaction was monitored by thin layer chromatography (TLC) using silica gel-G coated aluminium plates (0.5 mm thickness, Merck) and spots were visualized under UV radiation, purified by recrystallisation and column chromatography. The IR spectra were recorded on BRUKER TENSOR Series using KBr pellets. ${ }^{1} \mathrm{H}$ NMR spectra were recorded on $300 \mathrm{MHz}$ BRUKER ULTRASHIELD using DMSO- $\mathrm{d}_{6}$ as a solvent and TMS as an internal reference and chemical shift values were expressed in $\delta \mathrm{ppm}$.

\section{Chemistry}

The synthesis of the series proceeded as depicted in Schemes 1. 2-(Coumarinyl-4-oxy)-4(cyclopropylamino)-6-(aryl ureido)-s-triazine (5a-j) and 2-(Coumarinyl-4-oxy)-4(cyclopropylamino)-6-(aryl thioureido)-s-triazine (6a-j) were prepared in four steps from striazine. Briefly, s-triazine (1) was reacted with 4-hydroxy coumarin in presence of $\mathrm{Na}_{2} \mathrm{CO}_{3}$ to give 2-(Coumarinyl-4-oxy)-4,6-dichloro-s-triazine (2). The structure of compound (2) was confirmed by the IR and ${ }^{1} \mathrm{H}$ NMR spectral analysis. It is interesting to note that in IR spectra two sharp bands in the region of $1230,1040 \mathrm{~cm}^{-1}$ due to symmetrical and unsymmetrical C-O-C 
group respectively. Compound (2) was condensed with cyclopropyl to give 2-(Coumarinyl-4oxy)-4-(cyclopropylamino)-6-chloro-s-triazine (3). The IR spectra of compound (3) shown one sharp absorption band at $3315 \mathrm{~cm}^{-1}$ for $-\mathrm{NH}$ group and PNMR spectra of compound (3) shown singlet at $\delta 3.97$ of $-\mathrm{N} \underline{H}$ proton. Compound (4) was obtained by the condensation of compound (3) with ammonia. The structure of compound (4) was confirmed by the IR and ${ }^{1} \mathrm{H}$ NMR spectral analysis. Compound (4) was condensed with various isocyanate and isothiocyanate to afforded (5a-j) and (6a-j) respectively. All the synthesized compounds were fully characterized by IR, H-NMR spectroscopy and elemental analysis.

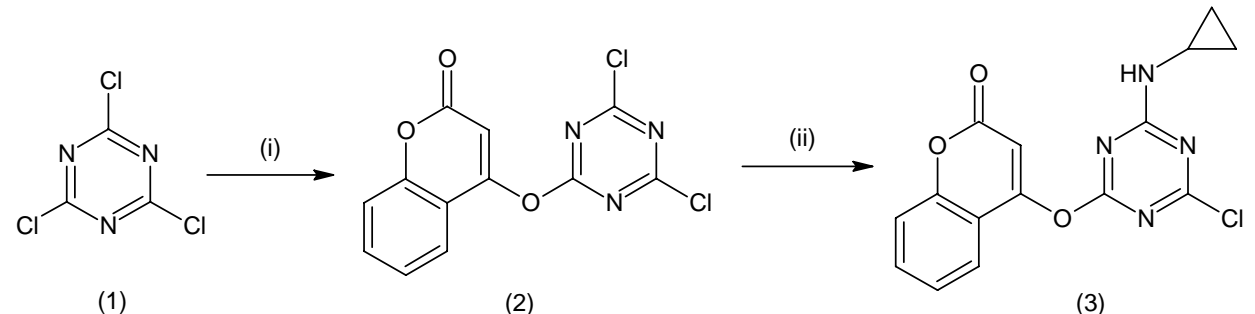

(1)

(2)
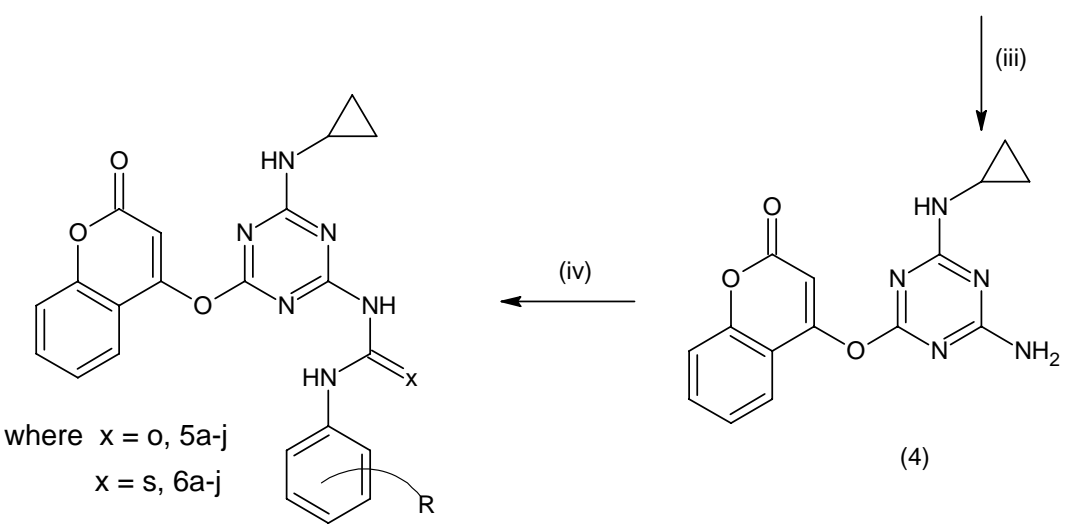

(4)

Scheme 1. Synthesis of the titled compounds. (i) 4-Hydroxy coumarin, Acetone, $\mathrm{Na}_{2} \mathrm{CO}_{3}$ (aq) (10\%), $0-5^{\circ} \mathrm{C}$, (ii) Cyclopropylamine, Acetone, $\mathrm{Na}_{2} \mathrm{CO}_{3}$ (aq) $(10 \%), 30-45^{\circ} \mathrm{C}$ (iii) Ammonia, $1,4-$ dioxane, $70-80^{\circ} \mathrm{C}$ (iv) Ar-NCX, Ethanol, reflux.

\section{Experimental Section}

2-(Coumarinyl-4-oxy)-4,6-dichloro-s-triazine (2). To a stirred solution of cyanuric chloride (1) $(4 \mathrm{~g}, 0.021 \mathrm{~mol})$ in acetone $(50 \mathrm{~mL})$ at $0-5^{\circ} \mathrm{C}$, the solution of 4-hydroxy coumarin $(3.5 \mathrm{~g}, 0.021$ mol) in $10 \% \mathrm{Na}_{2} \mathrm{CO}_{3}(25 \mathrm{~mL})$ was added drop wise in 1 hour. The stirring was continued at 0 $5^{\circ} \mathrm{C}$ for 4 hours. The progress of reaction was monitored by TLC using toluene: methanol (7:3) as eluent. After the completion of reaction, the content was poured into crushed ice water. Product was filtered, washed with cold water and dried to give (2), yield $78 \%, \mathrm{mp} 208-210^{\circ} \mathrm{C}$. 
2-(Coumarinyl-4-oxy)-4-(cyclopropylamino)-6-chloro-s-triazine (3). To a stirred solution of 2 $(4 \mathrm{~g}, 0.013 \mathrm{~mol})$ in acetone $(50 \mathrm{~mL})$ at $30-45^{\circ} \mathrm{C}$, cyclopropylamine $(0.74 \mathrm{~g}, 0.9 \mathrm{~mL}, 0.013 \mathrm{~mol})$ was added drop wise. The $\mathrm{pH}$ was adjusted neutral by the addition of $10 \% \mathrm{Na}_{2} \mathrm{CO}_{3}$ solution. The temperature was gradually raised to $45^{\circ} \mathrm{C}$ during 2 hours and further maintained for 2 hours. The progress of reaction was monitored by TLC using toluene: acetone (6:4) as eluent. After the completion of reaction, the content was poured into crushed ice. Product was filtered, washed with cold water and recrystallized from ethyl acetate to give (3) yield $60 \%, \mathrm{mp} 198-200^{\circ} \mathrm{C}$.

2-(Coumarinyl-4-oxy)-4-(cyclopropylamino)-6-(amino)-s-triazine (4). A mixture of (3) (3 g, $0.009 \mathrm{~mol})$ and $28 \%$ aqueous ammonia $(1.2 \mathrm{~mL}, 0.018 \mathrm{~mol})$ in 1 , 4-dioxane $(40 \mathrm{~mL})$ was heated at $70-80^{\circ} \mathrm{C}$ on water bath for 5 hours. The progress of reaction was monitored by TLC using toluene: acetone (5:5) as eluent. After the completion of reaction, the content was poured into ice water. Product was filtered, washed with cold water and recrystallized from ethanol to give (4) yield $65 \%$, mp $178-182^{\circ} \mathrm{C}$.

General procedure for the preparation of 2-(Coumarinyl-4-oxy)-4-(cyclopropylamino)-6(aryl ureido)-s-triazine (5a-j)

A mixture of (4) $(1.5 \mathrm{~g}, 0.005 \mathrm{~mol})$ and aryl isocyanate $(0.005 \mathrm{~mol})$ in ethanol $(30 \mathrm{~mL})$ was refluxed for 5-6 hours. The progress of reaction was monitored by TLC using toluene: acetone (7:3) as eluent. After the completion of reaction, the solvent was removed by distillation and the resulting solid was recrystallized from methanol.

2-(Coumarinyl-4-oxy)-4-(cyclopropylamino)-6-(phenyl ureido)-s-triazine (5a). Yield 64\%, mp 122-124 ${ }^{\circ} \mathrm{C}$. Anal. Calcd for $\mathrm{C}_{22} \mathrm{H}_{18} \mathrm{O}_{4} \mathrm{~N}_{6}$, Calculeted: $\mathrm{C}$ 61.39, H 4.22, N 19.53, Found : C 61.33, H 4.21, N 19.51. IR(KBr)cm ${ }^{-1}, 3315(\mathrm{~N}-\mathrm{H}$ str $), 1660(\mathrm{C}=\mathrm{O}$ urea $), 1340(\mathrm{C}-\mathrm{O}-\mathrm{C}), 1730(\mathrm{C}=\mathrm{O}$ $\delta$-lactone), 825(C-N), 2990(C-H). ${ }^{1} \mathrm{H}$ NMR (DMSO-d 6 , $\left.\delta\right)$ ppm, 0.49-0.63(m, 4H, $\left.2 \mathrm{CH}_{2}\right), 1.24-$ 1.53(m, 1H, CH), 3.97(s, 1H, NH), 5.97(s, 1H, Ar-H), 6.85(d, 1H, Ar-H), 7.12-8.0(m, 8H, ArH), 8.72(s, 1H, Ar-NHCO), 9.67(s,1H, CONH).

2-(Coumarinyl-4-oxy)-4-(cyclopropylamino)-6-(2-chloro penyl ureido)-s-triazine (5b). Yield $70 \%$, mp 242-244 ${ }^{\circ} \mathrm{C}$, Anal. Calcd for $\mathrm{C}_{22} \mathrm{H}_{17} \mathrm{O}_{4} \mathrm{~N}_{6} \mathrm{Cl}$, Calculeted : C 56.84, H 3.69, N 18.08, Found : C 56.81, H 3.68, N 18.05. IR(KBr) cm $\mathrm{cm}^{-1}$, 3255(N-H str), 1685(C=O urea), 1355(C-O-C), $1720(\mathrm{C}=\mathrm{O} \delta$-lactone $), 770\left(\mathrm{C}-\mathrm{Cl}\right.$ str), 835(C-N), 3010(C-H). ${ }^{1} \mathrm{H}$ NMR $\left(\mathrm{DMSO}_{6}, \delta\right) \mathrm{ppm}, 0.48-$ 0.61(m, 4H, $\left.2 \mathrm{CH}_{2}\right), 1.22-1.51(\mathrm{~m}, 1 \mathrm{H}, \mathrm{CH}), 3.92(\mathrm{~s}, 1 \mathrm{H}, \mathrm{NH}), 5.89(\mathrm{~s}, 1 \mathrm{H}, \mathrm{Ar}-\mathrm{H}), 6.7-7.9(\mathrm{~m}, 8 \mathrm{H}$, Ar-H), 8.79(s, 1H, Ar-NHCO), 9.54(s,1H, CONH).

2-(Coumarinyl-4-oxy)-4-(cyclopropylamino)-6-(3-chloro phenyl ureido)-s-triazine (5c). Yield 55\%, mp $162-164^{\circ} \mathrm{C}$, Anal. Calcd for $\mathrm{C}_{22} \mathrm{H}_{17} \mathrm{O}_{4} \mathrm{~N}_{6} \mathrm{Cl}$, Calculeted : C 56.84, $\mathrm{H} \mathrm{3.69,} \mathrm{N}$ 18.08, Found : C 56.79, H 3.72, N 18.03. IR(KBr) $\mathrm{cm}^{-1}, 3160(\mathrm{~N}-\mathrm{H}$ str), $1645(\mathrm{C}=\mathrm{O}$ urea),

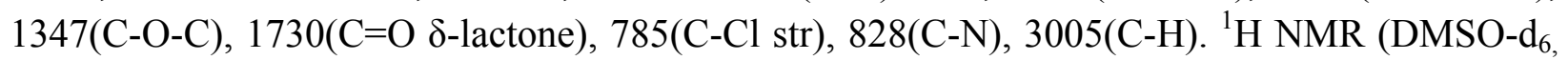

б) $\mathrm{ppm}, 0.50-0.66\left(\mathrm{~m}, 1 \mathrm{H}, 2 \mathrm{CH}_{2}\right), 1.30-1.58(\mathrm{~m}, 1 \mathrm{H}, \mathrm{CH}), 3.91(\mathrm{~s}, 1 \mathrm{H}, \mathrm{NH}), 5.80(\mathrm{~s}, 1 \mathrm{H}, \mathrm{Ar}-\mathrm{H})$, 6.61-7.8(m, 8H, Ar-H), 9.20(s, 1H, Ar-NHCO), 9.72(s,1H, CONH).

2-(Coumarinyl-4-oxy)-4-(cyclopropylamino)-6-(4-chloro phenyl ureido)-s-triazine (5d). Yield 59\%, mp $172-174^{\circ} \mathrm{C}$, Anal. Calcd for $\mathrm{C}_{22} \mathrm{H}_{17} \mathrm{O}_{4} \mathrm{~N}_{6} \mathrm{Cl}$, Calculeted : C 56.84, H 3.69, N 
18.08, Found : C 56.81, H 3.66, N 18.04. IR(KBr) $\mathrm{cm}^{-1}, 3250(\mathrm{~N}-\mathrm{H}$ str), $1712(\mathrm{C}=\mathrm{O}$ urea), 1352(C-O-C), 1732(C=O $\delta$-lactone), 690(C-Cl str), 825(C-N), 3028(C-H). ${ }^{1} \mathrm{H}$ NMR (DMSO-d 6 ,

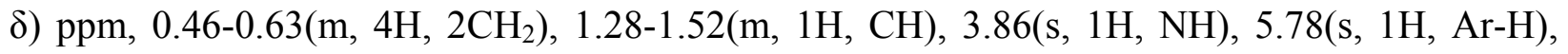
6.48(d, 2H, Ar-H), 6.9-7.84(m, 6H, Ar-H), 8.75(s, 1H, Ar-NHCO), 9.40(s,1H, CONH).

2-(Coumarinyl-4-oxy)-4-(cyclopropylamino)-6-(2-methyl phenyl ureido)-s-triazine (5e). Yield 64\%, mp 158-162 ${ }^{\circ} \mathrm{C}$, Anal. Calcd for $\mathrm{C}_{23} \mathrm{H}_{20} \mathrm{O}_{4} \mathrm{~N}_{6}$, Calculeted : C 62.36, H 4.54, N 18.90, Found : C 62.32, H 4.51, N 18.86. IR(KBr) cm $\mathrm{cm}^{-1}$, 3287(N-H str), 1694(C=O urea), 1335(C-O-C), $1740\left(\mathrm{C}=\mathrm{O} \delta\right.$-lactone), 832(C-N), 3025(C-H). ${ }^{1} \mathrm{H}$ NMR (DMSO- $\left.\mathrm{d}_{6}, \delta\right) \mathrm{ppm}, 0.48-0.64(\mathrm{~m}, 4 \mathrm{H}$, $\left.2 \mathrm{CH}_{2}\right), 1.27-1.49(\mathrm{~m}, 1 \mathrm{H}, \mathrm{CH}), 3.82(\mathrm{~s}, 1 \mathrm{H}, \mathrm{NH}), 5.87(\mathrm{~s}, 1 \mathrm{H}, \mathrm{Ar}-\mathrm{H}), 2.24\left(3 \mathrm{H}, \mathrm{s}, \mathrm{Ar}-\mathrm{CH}_{3}\right), 6.84-$ 7.82(m, 8H, Ar-H), 8.95(s, 1H, Ar-NHCO), 9.62(s,1H, CONH).

2-(Coumarinyl-4-oxy)-4-(cyclopropylamino)-6-(3-methyl phenyl ureido)-s-triazine (5f). Yield 62\%, mp 126-128 ${ }^{\circ} \mathrm{C}$, Anal. Calcd for $\mathrm{C}_{23} \mathrm{H}_{20} \mathrm{O}_{4} \mathrm{~N}_{6}$, Calculeted : C 62.36, H 4.54, N 18.90, Found : C 62.12, H 4.52, N 18.87. IR(KBr) cm $\mathrm{cm}^{-1}, 3298(\mathrm{~N}-\mathrm{H}$ str), 1705(C=O urea), 1330(C-O-C), $1755\left(\mathrm{C}=\mathrm{O} \delta\right.$-lactone), 818(C-N), 3037(C-H). ${ }^{1} \mathrm{H}$ NMR (DMSO-d 6 , $\left.\delta\right)$ ppm, 0.46-0.67(m, 4H, $\left.2 \mathrm{CH}_{2}\right), 1.25-1.46(\mathrm{~m}, 1 \mathrm{H}, \mathrm{CH}), 3.88(\mathrm{~s}, 1 \mathrm{H}, \mathrm{NH}), 5.94(\mathrm{~s}, 1 \mathrm{H}, \mathrm{Ar}-\mathrm{H}), 2.35\left(3 \mathrm{H}, \mathrm{s}, \mathrm{Ar}-\mathrm{CH}_{3}\right), 6.57(\mathrm{~s}$, 1H, Ar-H), 6.9-7.9(m, 7H, Ar-H), 8.82(s, 1H, Ar-NHCO), 9.58(s,1H, CONH).

2-(Coumarinyl-4-oxy)-4-(cyclopropylamino)-6-(4-methyl phenyl ureido)-s-triazine (5g). Yield 68\%, mp 112-114 ${ }^{\circ} \mathrm{C}$, Anal. Calcd for $\mathrm{C}_{23} \mathrm{H}_{20} \mathrm{O}_{4} \mathrm{~N}_{6}$, Calculeted : C 62.36, H 4.54, N 18.90, Found : C 62.12, H 4.52, N 18.88. IR(KBr) cm $\mathrm{cm}^{-1}$, 3257(N-H str), 1710(C=O urea), 1364(C-O-C), $1728\left(\mathrm{C}=\mathrm{O} \delta\right.$-lactone), 810(C-N), 3009(C-H). ${ }^{1} \mathrm{H}$ NMR (DMSO-d 6 , $\left.\delta\right)$ ppm, 0.48-0.68(m, 4H, $\left.2 \mathrm{CH}_{2}\right), 1.23-1.42(\mathrm{~m}, 1 \mathrm{H}, \mathrm{CH}), 3.92(\mathrm{~s}, 1 \mathrm{H}, \mathrm{NH}), 5.78(\mathrm{~s}, 1 \mathrm{H}, \mathrm{Ar}-\mathrm{H}), 2.23\left(\mathrm{~s}, 3 \mathrm{H}, \mathrm{Ar}-\mathrm{CH}_{3}\right), 6.57(\mathrm{~d}$, 2H, Ar-H), 7.08(d, 2H, Ar-H), 7.30-7.74(m, 4H, Ar-H), 9.02(s, 1H, Ar-NHCO), 9.47(s,1H, $\mathrm{CONH})$.

2-(Coumarinyl-4-oxy)-4-(cyclopropylamino)-6-(2-methoxy phenyl ureido)-s-triazine (5h). Yield $72 \%$, mp $172-174^{\circ} \mathrm{C}$, Anal. Calcd for $\mathrm{C}_{23} \mathrm{H}_{20} \mathrm{O}_{5} \mathrm{~N}_{6}$, Calculeted : C 61.39, H 4.38, N 18.25, Found : C 61.34, H 4.35, N 18.22. IR(KBr) cm $\mathrm{cm}^{-1}$, 3320(N-H str), 1698(C=O urea), 1305(C-O-C), 1742( $\mathrm{C}=\mathrm{O} \delta$ - lactone), 842(C-N), 3048(C-H). ${ }^{1} \mathrm{H}$ NMR (DMSO-d 6 , $\left.\delta\right)$ ppm, 0.50-0.70(m, 4H, $\left.2 \mathrm{CH}_{2}\right), 1.21-1.54(\mathrm{~m}, 1 \mathrm{H}, \mathrm{CH}), 3.95(\mathrm{~s}, 1 \mathrm{H}, \mathrm{NH}), 5.85(\mathrm{~s}, 1 \mathrm{H}, \mathrm{Ar}-\mathrm{H}), 3.48\left(3 \mathrm{H}, \mathrm{s}, \mathrm{Ar}-\mathrm{OCH}_{3}\right), 6.9-$ 8.1(m, 8H, Ar-H), 9.30(s, 1H, Ar-NHCO), 9.51(s,1H, CONH).

2-(Coumarinyl-4-oxy)-4-(cyclopropylamino)-6-(4-methoxy phenyl ureido)-s-triazine (5i). Yield 68\%, mp 186-188 ${ }^{\circ} \mathrm{C}$, Anal. Calcd for $\mathrm{C}_{23} \mathrm{H}_{20} \mathrm{O}_{5} \mathrm{~N}_{6}$, Calculeted : C 61.39, H 4.38, N 18.25, Found : C 61.37, H 4.36, N 18.23. IR(KBr) $\mathrm{cm}^{-1}$, 3322(N-H str), 1695(C=O urea), 1380(C-O-C), $1720\left(\mathrm{C}=\mathrm{O} \delta\right.$ - lactone), 845(C-N), 3072(C-H). ${ }^{1} \mathrm{H}$ NMR $\left(\mathrm{DMSO}_{6}, \delta\right) \mathrm{ppm}, 0.51-0.74(\mathrm{~m}, 4 \mathrm{H}$, $\left.2 \mathrm{CH}_{2}\right), 1.25-1.63(\mathrm{~m}, 1 \mathrm{H}, \mathrm{CH}), 4.21(\mathrm{~s}, 1 \mathrm{H}, \mathrm{NH}), 5.69(\mathrm{~s}, 1 \mathrm{H}, \mathrm{Ar}-\mathrm{H}), 3.87\left(3 \mathrm{H}, \mathrm{s}, \mathrm{Ar}-\mathrm{OCH}_{3}\right)$, 6.44(d, 2H, Ar-H), 7.28(d, 2H, Ar-H), 7.43-7.77(m, 6H, Ar-H), 9.18(s, 1H, Ar-NHCO), 9.62(s,1H, CONH).

2-(Coumarinyl-4-oxy)-4-(cyclopropylamino)-6-(3-nitro phenyl ureido)-s-triazine (5j). Yield $56 \%$, mp 88-90 ${ }^{\circ} \mathrm{C}$, Anal. Calcd for $\mathrm{C}_{22} \mathrm{H}_{17} \mathrm{O}_{6} \mathrm{~N}_{7}$, Calculeted : C 55.58, H 3.60, N 20.62, Found : $\mathrm{C} 55.55, \mathrm{H} 3.57, \mathrm{~N}$ 20.58. $\mathrm{IR}(\mathrm{KBr}) \mathrm{cm}^{-1}, 3280(\mathrm{~N}-\mathrm{H}$ str $), 1660(\mathrm{C}=\mathrm{O}$ urea $), 1358(\mathrm{C}-\mathrm{O}-\mathrm{C})$, $1736(\mathrm{C}=\mathrm{O} \delta$ - lactone $), 890(\mathrm{C}-\mathrm{N}), 1527(\mathrm{~N}=\mathrm{O}), 3040(\mathrm{C}-\mathrm{H}) .{ }^{1} \mathrm{H}$ NMR $\left(\mathrm{DMSO}_{6}, \delta\right) \mathrm{ppm}, 0.44-$ 
0.56(m, 4H, 2CH 2$), 1.24-1.41(\mathrm{~s}, 1 \mathrm{H}, \mathrm{CH}), 4.0(\mathrm{~s}, 1 \mathrm{H}, \mathrm{NH}), 5.64(\mathrm{~s}, 1 \mathrm{H}, \mathrm{Ar}-\mathrm{H}), 8.39(\mathrm{~s}, 1 \mathrm{H}, \mathrm{Ar}-\mathrm{H})$, 7.0-8.1(m, 7H, Ar-H), 8.72(s, 1H, Ar-NHCO), 9.25(s,1H, CONH).

General procedure for the preparation of 2-(Coumarinyl-4-oxy)-4-(cyclopropylamino)-6(phenyl thioureido)-s-triazine (6a-j)

A mixture of (4) $(1.5 \mathrm{~g}, 0.005 \mathrm{~mol})$ and aryl isothiocyanate $(0.005 \mathrm{~mol})$ in ethanol $(30 \mathrm{~mL})$ was refluxed for 5-6 hours. The progress of reaction was monitored by TLC using toluene: acetone (6:4) as eluent. After the completion of reaction, the solvent was removed by distillation and the resulting solid was recrystallized from methanol.

2-(Coumarinyl-4-oxy)-4-(cyclopropylamino)-6-(phenyl thioureido)-s-triazine (6a). Yield $72 \%, \mathrm{mp} \mathrm{80-82}{ }^{\circ} \mathrm{C}$, Anal. Calcd for $\mathrm{C}_{22} \mathrm{H}_{18} \mathrm{O}_{3} \mathrm{~N}_{6} \mathrm{~S}$, Calculeted : C 59.18, H 4.02, N 18.82, Found : C 59.15, H 4.01, N 18.79. IR(KBr) cm ${ }^{-1}, 3246(\mathrm{~N}-\mathrm{H}$ str), $1400(\mathrm{C}=\mathrm{S}), 1265(\mathrm{C}-\mathrm{O}-\mathrm{C}), 1699(\mathrm{C}=\mathrm{O} \delta-$ lactone), 785(C-N), 2992(C-H). ${ }^{1} \mathrm{H}$ NMR (DMSO-d 6 $\left.\delta\right)$ ppm, 0.30-0.54(m, 4H, 2CH $), 1.22-$ 1.50(s, 1H, CH), 3.8(s, 1H, NH), 5.72(s, 1H, Ar-H), 6.8(dd, 1H, Ar-H), 7.2-7.9(m, 7H, Ar-H), 10.9(s, 1H, Ar-NHCS), 11.18(s, 1H, CSNH).

2-(Coumarinyl-4-oxy)-4-(cyclopropylamino)-6-(2-chloro phenyl thioureido)-s-triazine (6b). Yield 66\%, mp 94-96 ${ }^{\circ} \mathrm{C}$, Anal. Calcd for $\mathrm{C}_{22} \mathrm{H}_{17} \mathrm{O}_{3} \mathrm{~N}_{6} \mathrm{SCl}$, Calculeted : C 54.94, H 3.56, N 17.47, Found : C 54.91, H 3.52, N 17.44. IR(KBr) cm ${ }^{-1}, 3285(\mathrm{~N}-\mathrm{H}$ str), 1440(C=S), 1204(C-O-C), $1735\left(\mathrm{C}=\mathrm{O} \delta\right.$-lactone), 810(C-N), 3045(C-H). ${ }^{1} \mathrm{H}$ NMR (DMSO- $\left.\mathrm{d}_{6} \delta\right) \mathrm{ppm}, 0.41-0.56(\mathrm{~m}, 4 \mathrm{H}$, $\left.2 \mathrm{CH}_{2}\right), 1.20-1.48(\mathrm{~m}, 1 \mathrm{H}, \mathrm{CH}), 4.1(\mathrm{~s}, 1 \mathrm{H}, \mathrm{NH}), 5.86(\mathrm{~s}, 1 \mathrm{H}, \mathrm{Ar}-\mathrm{H}), 6.8-7.89(\mathrm{~m}, 8 \mathrm{H}, \mathrm{Ar}-\mathrm{H})$, 11.01(s, 1H, Ar-NHCS), 11.4(s, 1H, CSNH).

2-(Coumarinyl-4-oxy)-4-(cyclopropylamino)-6-(3-chloro phenyl thioureido)-s-triazine (6c). Yield 59\%, mp 92-94 ${ }^{\circ} \mathrm{C}$, Anal. Calcd for $\mathrm{C}_{22} \mathrm{H}_{17} \mathrm{O}_{3} \mathrm{~N}_{6} \mathrm{SCl}$, Calculeted : C 54.94, H 3.56, N 17.47, Found : C 54.91, H 3.52, N 17.44. IR(KBr) cm $\mathrm{cm}^{-1}$ 3318(N-H str), 1510(C=S), 1228(C-O-C), $1750\left(\mathrm{C}=\mathrm{O} \delta\right.$-lactone), $880(\mathrm{C}-\mathrm{N}), 3025(\mathrm{C}-\mathrm{H}) .{ }^{1} \mathrm{H}$ NMR (DMSO- $\left.\mathrm{d}_{6} \delta\right) \mathrm{ppm}, 0.37-0.54(\mathrm{~m}, 4 \mathrm{H}$, $\left.2 \mathrm{CH}_{2}\right), 1.23-1.51(\mathrm{~m}, 1 \mathrm{H}, \mathrm{CH}), 3.8(\mathrm{~s}, 1 \mathrm{H}, \mathrm{NH}), 5.91(\mathrm{~s}, 1 \mathrm{H}, \mathrm{Ar}-\mathrm{H}), 6.69(1 \mathrm{H}, \mathrm{s}, \mathrm{Ar}-\mathrm{H}), 7.1-8.2(\mathrm{~m}$, 7H, Ar-H), 10.87(s, 1H, Ar-NHCS), 11.25(s, 1H, CSNH).

2-(Coumarinyl-4-oxy)-4-(cyclopropylamino)-6-(4-chloro phenyl thioureido)-s-triazine (6d). Yield 63\%, mp 74-76 ${ }^{\circ} \mathrm{C}$, Anal. Calcd for $\mathrm{C}_{22} \mathrm{H}_{17} \mathrm{O}_{3} \mathrm{~N}_{6} \mathrm{SCl}$, Calculeted : C 54.94, H 3.56, N 17.47, Found : C 54.92, H 3.51, N 17.44. IR(KBr) cm ${ }^{-1}, 3280(\mathrm{~N}-\mathrm{H}$ str), 1435(C=S), 1205(C-O-C), 1698(C=O $\delta$-lactone), 852(C-N), 3060(C-H). ${ }^{1} \mathrm{H}$ NMR (DMSO- $\left.\mathrm{d}_{6} \delta\right) \mathrm{ppm}, 0.39-0.53(\mathrm{~m}, 4 \mathrm{H}$, $\left.2 \mathrm{CH}_{2}\right), 1.23-1.35(\mathrm{~s}, 1 \mathrm{H}, \mathrm{CH}), 3.97(\mathrm{~s}, 1 \mathrm{H}, \mathrm{NH}), 5.79(\mathrm{~s}, 1 \mathrm{H}, \mathrm{Ar}-\mathrm{H}), 6.58(\mathrm{~d}, 2 \mathrm{H}, \mathrm{Ar}-\mathrm{H}), 7.38(\mathrm{~d}, 2 \mathrm{H}$, Ar-H), 7.50-7.64(m, 4H, Ar-H) 10.87(s, 1H, Ar-NHCS), 11.09(s, 1H, CSNH).

2-(Coumarinyl-4-oxy)-4-(cyclopropylamino)-6-(2-methyl phenyl thioureido)-s-triazine (6e). Yield 69\%, mp 102-104 ${ }^{\circ} \mathrm{C}$, Anal. Calcd for $\mathrm{C}_{23} \mathrm{H}_{20} \mathrm{O}_{3} \mathrm{~N}_{6} \mathrm{~S}$, Calculeted : C 59.99, H 4.38, N 18.25, Found : C 59.94, H 4.36, N 18.21. IR(KBr) cm $\mathrm{cm}^{-1}, 3318(\mathrm{~N}-\mathrm{H}$ str), 1510(C=S), 1225(C-O-C), $1722\left(\mathrm{C}=\mathrm{O} \delta\right.$-lactone), $870(\mathrm{C}-\mathrm{N}), 3076(\mathrm{C}-\mathrm{H}) .{ }^{1} \mathrm{H}$ NMR (DMSO- $\left.\mathrm{d}_{6} \delta\right) \mathrm{ppm}, 0.37-0.52(\mathrm{~m}, 4 \mathrm{H}$, $\left.2 \mathrm{CH}_{2}\right), 1.22-1.52(\mathrm{~s}, 1 \mathrm{H}, \mathrm{CH}), 4.0(\mathrm{~s}, 1 \mathrm{H}, \mathrm{NH}), 5.82(\mathrm{~s}, 1 \mathrm{H}, \mathrm{Ar}-\mathrm{H}), 2.3\left(\mathrm{~s}, 3 \mathrm{H}, \mathrm{Ar}_{-} \mathrm{CH}_{3}\right), 6.8-7.92(\mathrm{~m}$, $8 \mathrm{H}, \mathrm{Ar}-\mathrm{H}), 10.72(\mathrm{~s}, 1 \mathrm{H}, \mathrm{Ar}-\mathrm{NHCS}), 11.2(\mathrm{~s}, 1 \mathrm{H}, \mathrm{CSNH})$. 
2-(Coumarinyl-4-oxy)-4-(cyclopropylamino)-6-(3-methyl phenyl thioureido)-s-triazine (6f). Yield 58\%, mp 114-116 ${ }^{\circ} \mathrm{C}$, Anal. Calcd for $\mathrm{C}_{23} \mathrm{H}_{20} \mathrm{O}_{3} \mathrm{~N}_{6} \mathrm{~S}$, Calculeted : C 59.99, H 4.38, N 18.25, Found : C 59.97, H 4.34, N 18.21. IR(KBr) cm $\mathrm{cm}^{-1}$, 3257(N-H str), 1350(C=S), 1272(C-O-C), $1706\left(\mathrm{C}=\mathrm{O} \delta\right.$-lactone), 856(C-N), 3012(C-H). ${ }^{1} \mathrm{H}$ NMR (DMSO- $\left.\mathrm{d}_{6} \delta\right)$ ppm, 0.38-0.53(m, 4H, $\left.2 \mathrm{CH}_{2}\right), 1.21-1.52(\mathrm{~m}, 1 \mathrm{H}, \mathrm{CH}), 3.97(\mathrm{~s}, 1 \mathrm{H}, \mathrm{NH}), 5.82(\mathrm{~s}, 1 \mathrm{H}, \mathrm{Ar}-\mathrm{H}), 2.13\left(\mathrm{~s}, 3 \mathrm{H}, \mathrm{Ar}-\mathrm{CH}_{3}\right), 6.58(\mathrm{~s}$, 1H, Ar-H), 6.9-8.0(m, 7H, Ar-H), 10.9(s, 1H, Ar-NHCS), 11.42(s, 1H, CSNH).

2-(Coumarinyl-4-oxy)-4-(cyclopropylamino)-6-(4-methyl phenyl thioureido)-s-triazine (6g). Yield $65 \%$, mp $78-80^{\circ} \mathrm{C}$, Anal. Calcd for $\mathrm{C}_{23} \mathrm{H}_{20} \mathrm{O}_{3} \mathrm{~N}_{6} \mathrm{~S}$, Calculeted : C 59.99, H 4.38, N 18.25, Found : C 59.96, H 4.34, N 18.21. IR(KBr) cm ${ }^{-1}$, 3362(N-H str), 1490(C=S), 1198(C-O-C), 1688(C=O $\delta$-lactone), 905(C-N), 3040(C-H). ${ }^{1} \mathrm{H}$ NMR (DMSO-d 6 $\left.\delta\right)$ ppm, 0.4-0.54(m, 4H, $\left.2 \mathrm{CH}_{2}\right), 1.2-1.5(\mathrm{~m}, 1 \mathrm{H}, \mathrm{CH}), 3.72(\mathrm{~s}, 1 \mathrm{H}, \mathrm{NH}), 6.0(\mathrm{~s}, 1 \mathrm{H}, \mathrm{Ar}-\mathrm{H}), 2.3\left(\mathrm{~s}, 3 \mathrm{H}, \mathrm{Ar}-\mathrm{CH}_{3}\right), 6.49(\mathrm{~d}, 2 \mathrm{H}$, Ar-H), 6.55(d, 2H, Ar-H), 6.8-7.9(m, 4H, Ar-H), 10.8(s, 1H, Ar-NHCS), 11.3(s, 1H, CSNH).

2-(Coumarinyl-4-oxy)-4-(cyclopropylamino)-6-(2-methoxy phenyl thioureido)-s-triazine (6h). Yield 70\%, mp 102-104 ${ }^{\circ} \mathrm{C}$, Anal. Calcd for $\mathrm{C}_{23} \mathrm{H}_{20} \mathrm{O}_{4} \mathrm{~N}_{6} \mathrm{~S}$, Calculeted : C 57.97, H 4.23, N 17.64, Found : C 57.95, H 4.22, N 17.61. IR(KBr) cm $\mathrm{cm}^{-1}, 3300(\mathrm{~N}-\mathrm{H}$ str), $1510(\mathrm{C}=\mathrm{S}), 1140(\mathrm{C}-\mathrm{O}-$ C), $1725\left(\mathrm{C}=\mathrm{O} \delta\right.$-lactone), 835(C-N), 3020(C-H). ${ }^{1} \mathrm{H}$ NMR $\left(\mathrm{DMSO}_{6} \mathrm{~d}_{6} \delta\right) \mathrm{ppm}, 0.37-0.51(\mathrm{~m}, 4 \mathrm{H}$, $\left.2 \mathrm{CH}_{2}\right), 1.3-1.6(\mathrm{~m}, 1 \mathrm{H}, \mathrm{CH}), 3.92(\mathrm{~s}, 1 \mathrm{H}, \mathrm{NH}), 5.82(\mathrm{~s}, 1 \mathrm{H}, \mathrm{Ar}-\mathrm{H}), 3.32\left(3 \mathrm{H}, \mathrm{s}, \mathrm{Ar}-\mathrm{OCH}_{3}\right), 6.9-$ 8.1(m, 8H, Ar-H), 11.13(s, 1H, Ar-NHCS), 11.54(s, 1H, CSNH).

2-(Coumarinyl-4-oxy)-4-(cyclopropylamino)-6-(4-methoxy phenyl thioureido)-s-triazine (6i). Yield $74 \%$, mp $84-86^{\circ} \mathrm{C}$, Anal. Calcd for $\mathrm{C}_{23} \mathrm{H}_{20} \mathrm{O}_{4} \mathrm{~N}_{6} \mathrm{~S}$, Calculeted : C 57.97, H 4.23, N 17.64, Found : C 57.93, H 4.21, N 17.60. IR(KBr) cm $\mathrm{cm}^{-1}, 3310(\mathrm{~N}-\mathrm{H}$ str), 1425(C=S), $1190(\mathrm{C}-\mathrm{O}-$ C), $1704\left(\mathrm{C}=\mathrm{O} \delta\right.$-lactone), 892(C-N), 3075(C-H). ${ }^{1} \mathrm{H}$ NMR (DMSO-d 6 $\left.\delta\right)$ ppm, 0.47-0.53(m, 4H, $\left.2 \mathrm{CH}_{2}\right), 1.4-1.5(\mathrm{~m}, 1 \mathrm{H}, \mathrm{CH}), 3.82(\mathrm{~s}, 1 \mathrm{H}, \mathrm{NH}), 5.9(\mathrm{~s}, 1 \mathrm{H}, \mathrm{Ar}-\mathrm{H}), 3.42\left(\mathrm{~s}, 3 \mathrm{H}, \mathrm{Ar}-\mathrm{OCH}_{3}\right), 6.63(\mathrm{~d}$, $2 \mathrm{H}, \mathrm{Ar}-\mathrm{H}), 6.82(\mathrm{~d}, 2 \mathrm{H}, \mathrm{Ar}-\mathrm{H}), 7.0-8.1(\mathrm{~m}, 4 \mathrm{H}, \mathrm{Ar}-\mathrm{H}), 10.92(\mathrm{~s}, 1 \mathrm{H}, \mathrm{Ar}-\mathrm{NHCS}), 11.42(\mathrm{~s}, 1 \mathrm{H}$, $\mathrm{CSNH})$.

2-(Coumarinyl-4-oxy)-4-(cyclopropylamino)-6-(4-nitro phenyl thioureido)-s-triazine (6j). Yield 54\%, mp 86-88 ${ }^{\circ} \mathrm{C}$, Anal. Calcd for $\mathrm{C}_{23} \mathrm{H}_{17} \mathrm{O}_{5} \mathrm{~N}_{7} \mathrm{~S}$, Calculeted : C 53.76, H 3.49, N 19.95, Found : C 53.75, H 3.47, N 19.92. IR(KBr) cm ${ }^{-1}$, 3360(N-H str), 1470(C=S), 1218(C-O-C), $1710(\mathrm{C}=\mathrm{O} \delta$-lactone $), 878(\mathrm{C}-\mathrm{N}), 3060(\mathrm{C}-\mathrm{H}), \quad 1520(\mathrm{~N}=\mathrm{O}) .{ }^{1} \mathrm{H}$ NMR $\left(\mathrm{DMSO}_{\mathrm{d}} \mathrm{d}\right) \mathrm{ppm}, 0.49-$ 0.54(m, 4H, 2CH $)$, 1.3-1.47(m, 1H, CH), 3.94(s, 1H, NH), 5.76(s, 1H, Ar-H), 6.59(d, 2H, ArH), 6.9-7.8(m, 6H, Ar-H), 10.84(s, 1H, Ar-NHCS), 11.32(s, 1H, CSNH).

\section{Antibacterial activity}

The minimum inhibitory concentration (MIC) of the synthesized compound were tested against two representative Gram-positive (Staphylococcus aureus ATCC 25923 and Bacillus subtilis ATCC 6633) and two Gram-negative (Escherichia coli ATCC 25922 and Pseudomonas aeruginosa ATCC 27853) by broth dilution method recommended by National Committee for Clinical Laboratory (NCCL) standards ${ }^{20}$ with gradual dilution starting from $(100,50,25,12.5$, $6.25,3.125) \mu \mathrm{g} / \mathrm{mL}$. Penicillin and Streptomycin were used as reference antibacterial agents. Solution of the test compounds and reference drugs were dissolved in DMSO. 
Table 1. Antibacterial activities

\begin{tabular}{|c|c|c|c|c|c|c|}
\hline \multirow[b]{2}{*}{ Compound } & \multirow[b]{2}{*}{$\mathrm{R}$} & \multirow[b]{2}{*}{$X$} & \multicolumn{4}{|c|}{ Minimum Inhibitory Concentration in $\mu \mathrm{g} / \mathrm{mL}$} \\
\hline & & & $\begin{array}{c}\text { Staphylococcus } \\
\text { aureus }\end{array}$ & $\begin{array}{l}\text { Bacillus } \\
\text { subtilis }\end{array}$ & $\begin{array}{c}\text { Pseudomonas } \\
\text { aeruginosa }\end{array}$ & $\begin{array}{c}\text { Escherichia } \\
\text { coli }\end{array}$ \\
\hline $5 a$ & $\mathrm{H}$ & $\mathrm{O}$ & 25 & 50 & 100 & 25 \\
\hline $5 \mathbf{b}$ & $2-\mathrm{Cl}$ & $\mathrm{O}$ & 12.5 & 25 & 50 & 50 \\
\hline $5 c$ & $3-\mathrm{Cl}$ & $\mathrm{O}$ & 25 & 6.25 & - & 25 \\
\hline $5 d$ & $4-\mathrm{Cl}$ & $\mathrm{O}$ & 50 & 6.25 & 100 & 50 \\
\hline $5 e$ & $2-\mathrm{CH}_{3}$ & $\mathrm{O}$ & 100 & 25 & - & 100 \\
\hline $5 f$ & $3-\mathrm{CH}_{3}$ & $\mathrm{O}$ & 50 & 12.5 & 50 & 25 \\
\hline $5 g$ & $4-\mathrm{CH}_{3}$ & $\mathrm{O}$ & 12.5 & - & 100 & 50 \\
\hline $5 \mathrm{~h}$ & $2-\mathrm{OCH}_{3}$ & $\mathrm{O}$ & 12.5 & 25 & 50 & 12.5 \\
\hline $5 \mathbf{i}$ & $4-\mathrm{OCH}_{3}$ & $\mathrm{O}$ & 6.25 & 50 & 100 & 25 \\
\hline $5 \mathbf{j}$ & $3-\mathrm{NO}_{2}$ & $\mathrm{O}$ & 25 & 100 & - & 50 \\
\hline $6 a$ & $\mathrm{H}$ & $\mathrm{S}$ & 12.5 & 50 & 50 & 25 \\
\hline 6b & $2-\mathrm{Cl}$ & $\mathrm{S}$ & 25 & 100 & 100 & 50 \\
\hline 6c & $3-\mathrm{Cl}$ & $\mathrm{S}$ & 12.5 & 25 & 50 & 50 \\
\hline 6d & $4-\mathrm{Cl}$ & $\mathrm{S}$ & 25 & 6.25 & 100 & 12.5 \\
\hline $6 e$ & $2-\mathrm{CH}_{3}$ & $\mathrm{~S}$ & 50 & 100 & 50 & 25 \\
\hline $6 f$ & $3-\mathrm{CH}_{3}$ & $\mathrm{~S}$ & 6.25 & 25 & 25 & 12.5 \\
\hline $6 g$ & $4-\mathrm{CH}_{3}$ & $\mathrm{~S}$ & 12.5 & - & 12.5 & 50 \\
\hline $6 h$ & $2-\mathrm{OCH}_{3}$ & $\mathrm{~S}$ & 100 & 50 & 50 & 25 \\
\hline $6 \mathbf{i}$ & $4-\mathrm{OCH}_{3}$ & $\mathrm{~S}$ & 12.5 & 12.5 & 25 & 50 \\
\hline $6 \mathbf{j}$ & $4-\mathrm{NO}_{2}$ & $\mathrm{~S}$ & - & 12.5 & 100 & 25 \\
\hline Penicillin & & & 1.562 & 1.562 & 12.5 & 6.25 \\
\hline Streptomycin & & & 6.25 & 6.25 & 3.125 & 3.125 \\
\hline
\end{tabular}

For antibacterial activity, in present protocol $25 \mu \mathrm{g} \mathrm{mL}^{-1}$ is considered as moderate activity, 12.5 $\mu \mathrm{g} \mathrm{mL} L^{-1}$ is considered as good activity and $6.25 \mu \mathrm{g} \mathrm{mL}^{-1}$ is considered as excellent active compound to the standard drug.

\section{Results and Discussion}

The present paper is focused on the synthesis of novel heterocyclic compounds as possible antibacterial agents. The minimum inhibitory concentration (MICs, $\mu \mathrm{g} \mathrm{mL}^{-1}$ ) of tested compounds against bacteria are shown in Table I. All the synthesized compounds exhibited moderate to excellent inhibitory effect with MIC values against four strains of bacteria (Grampositive, Gram-negative). The ureido linkage two chloro substituted (5c and $\mathbf{5 d}$ ) and methoxy substituted (5i) compounds showed excellent activity against Gram-positive B. subtillus and $S$. 
aureus bacteria. Among the thioureido linkage compounds, (6d and 6f) displayed excellent activity against Gram-positive B. subtillis and S. aureus organism. In general, it is concluded that the presence of electron withdrawing group increases the antibacterial activities compare to the electron donating group to the aromatic ring. Based upon this observation it becomes necessary to optimize lead compound with chloro substitution for augmenting antibacterial prob. Based upon the results, it will also be necessary to optimize by the substituting a series of electronwithdrawing groups on aromatic ring and selectively modifying the s-triazine nucleus. The substitution in the C-3 and C-4 positions of the phenyl ring seems to be very important for antibacterial effect, as well as the presence and the position of oxo-linkage in the connecting linker between the aromatic rings seems to be very important for antibacterial effect.

\section{Acknowledgements}

Authors are thankful to Dr.U.C.Pande Head, Department of Chemistry \& Director, School of Sciences, Gujarat University, for his kind co-operation and Zydus Research Centre providing characterization data.

\section{References}

1. Garmouna, M.; Blanchoud, H.; Teil, M.; Blanchard, M.; Chevreuil, M. Water, Air, Soil, Poll. 2001, 132, 1.

2. Jain, S.; Bhambi, D.; Sharma, R.; Talesara, L. Ind. J. Pharm. Sci. 2007, 69(1), 28.

3. Klenke, B.; Stewart, M.; Barett, M. P.; Brun, R.; Gilbert, I. H. J. Med. Chem. 2001, 44, 3440.

4. Kukla, M. J.; Janseen, P. A. Eur. Pat. 1999, 945, 447.

5. Patel, R. B.; Chikhalia, K. H.; De clercq, E. J. Braz. Chem. Soc. 2007, 18, 2.

6. Srinivas, K.; Shrinivas, U.; Rao, V. J.; Bhanuprakash, K.; Chishore, K. H.; Murty, U. S. N. Bioorg. Med. Chem. Lett. 2005, 15, 1121.

7. Lubbers, T.; Angechm, P.; Gumunder, H.; Herzig, S.; Kulhanek, J. Bioorg. Chem. Lett. 2000, 3, 821 .

8. Desai, A. D.; Mahajan, D. H.; Chikhalia, K. H. Ind. J. Chem. 2007, 46B, 1169.

9. Srinivas, K.; Srinivas, U.; Bhanuprakash, K.; Harakishore, K.; Murthy, U. S. N.; Rao, V. J. Euro. J. Med. Chem. 2006, 41, 1240.

10. Alteramn, M.; Samulsson, B. J. Med. Chem. 1998, 41, 3782.

11. Ktritzky, A. R.; Boris, V. R.; Vladimir, Y. V.; Normand, H and Behranz, F. J. Org. Chem. 2001, 66, 6797.

12. Mayumi, O.; Kawahara, N. and Santo, Y. Cancer Res. 1996, 56, 1512.

13. Brozowski, Z.; Gdaniec, M. Eur. J. Med. Chem. 2000, 35, 1053. 
14. Shin-ichi, Y.; Yasuhisa, F.; Ichiro, K.; Hisashi, Y.; Toshiyuki, M.; Hiroaki, G.; Takato, Y. J. Nat. Cancer Inst. 2006, 98(8), 545.

15. Youngyuth, Y.; Tirayut, V.; Netnapa, C.; Sumalee, K.; Gordon, L. J. Med. Chem. 2000, 43, 2738.

16. Agrwal, A.; Srivastava, K.; Puri, S. K. and Chauhan, M. S. Bioorg. Med. Chem. Lett. 2005, 15, 531.

17. Irena, K.; Georgi, M.; Margarita, K. Bioorg. Chem. Appl. 2006, 1.

18. Jung, J. C.; Lee, J. H.; Oh, S.; Lee, J. G. Bioorg. Med. Chem. Lett. 2004, 14(22), 27.

19. Sueed, U. R.; Zahid, H. C.; Lunlaz, F.; Supuram, C. T. J. Enzy. Inh. Med. Chem. 2005, 20(4), 333.

20. National Committee for Clinical Laboratory standards (NCCLS). Standard methods for dilution antimicrobial susceptibility test for bacteria which grows aerobically Nat. Comm. Clini. Lab. Stands. Villanova. 1982, 242. 